\title{
SOME MODELS OF CAHN-HILLIARD EQUATIONS IN NONISOTROPIC MEDIA
}

\begin{abstract}
Alain Miranville ${ }^{1}$
Abstract. We derive in this article some models of Cahn-Hilliard equations in nonisotropic media. These models, based on constitutive equations introduced by Gurtin in [19], take the work of internal microforces and also the deformations of the material into account. We then study the existence and uniqueness of solutions and obtain the existence of finite dimensional attractors.

Résumé. Nous obtenons dans cet article des modèles d'équations de Cahn-Hilliard basés sur des équations constitutives introduites par Gurtin dans [19]. Ces modèles prennent en compte le travail de microforces internes ainsi que les déformations du matériau. Nous obtenons alors l'existence et l'unicité de solutions, puis l'existence d'attracteurs de dimension finie.
\end{abstract}

Mathematics Subject Classification. 35A05, 35B40, 35B45.

Received: June 14, 1999. Revised: October 1st, 1999.

\section{INTRODUCTION}

The Cahn-Hilliard equation (see [5] and [6]) is very central to material sciences. This equation, which is a conservation law, describes the transport of atoms between unit cells. It is based on a constitutive equation for the mass conservation of the form

$$
\frac{\partial \rho}{\partial t}=\kappa \Delta \mu, \kappa>0
$$

where $\rho$ is the order parameter (which corresponds to a density of atoms here) and $\mu$ is the chemical potential, and on a constitutive equation for the chemical potential of the form

$$
\mu=f^{\prime}(\rho)-\alpha \Delta \rho, \alpha>0,
$$

where $f$ is a double-well potential whose wells define the phases of the material. The potential $f$ is usually a polynomial of degree four (see $[5,6]$ and [30]); in this article, we consider more generally a polynomial of arbitrary even degree with strictly positive leading coefficient. We refer the reader to $[10-12,15,21]$ for other types of potentials; however, we cannot extend our results to such potentials.

Keywords and phrases. Cahn-Hilliard equation, internal microforces, deformable continuum, nonisotropic material, global attractor, exponential attractor.

1 Université de Poitiers, Mathématiques, SP2MI, Téléport 2, boulevard Marie et Pierre Curie, 86962 Chasseneuil Futuroscope Cedex, France. e-mail: miranv@wallis.sp2mi.univ-poitiers.fr 
In [19], Gurtin derived more general constitutive equations for the mass balance and the chemical potential (we refer the interested reader to [19] for a discussion on the objections that the author makes on the classical Cahn-Hilliard theory and which leads to the introduction of more complete constitutive equations).

Now, we describe the models that we wish to study. First, we consider a generalization of (1.1) which reads (see [19])

$$
\frac{\partial \rho}{\partial t}=\operatorname{div} B \nabla \mu+g
$$

where $B$ is a symmetric positive definite tensor with constant coefficients (more generally, the tensor $B$ may also depend on $\rho, \nabla \rho, \frac{\partial \rho}{\partial t}, \mu$ and $\nabla \mu$, in which case it may also degenerate) and where $g=g(x)$ is an external mass supply. Furthermore, by taking the work of internal microforces into account, Gurtin obtains a more general constitutive equation for the chemical potential of the form

$$
\mu-b . \nabla \mu=f^{\prime}(\rho)-\alpha \Delta \rho+\beta \frac{\partial \rho}{\partial t}-\gamma, \beta>0
$$

where $b$ is a constant vector which vanishes for isotropic materials (again, the vector $b$ may also depend on $\rho$, $\nabla \rho, \frac{\partial \rho}{\partial t}, \mu$ and $\left.\nabla \mu\right)$ and where $\gamma=\gamma(x)$ corresponds to the action of external microforces. We deduce from (1.4) that

$$
\operatorname{div} B \nabla \mu-b \cdot \nabla \operatorname{div} B \nabla \mu=\operatorname{div} B \nabla f^{\prime}(\rho)-\alpha \operatorname{div} B \nabla \rho+\beta \frac{\partial}{\partial t} \operatorname{div} B \nabla \rho-\operatorname{div} B \nabla \gamma,
$$

and we finally obtain, thanks to (1.3), the following generalized Cahn-Hilliard equation:

$$
\frac{\partial \rho}{\partial t}-\frac{\partial}{\partial t} b . \nabla \rho-\beta \frac{\partial}{\partial t} \operatorname{div} B \nabla \rho+\alpha \operatorname{div} B \nabla \Delta \rho-\operatorname{div} B \nabla f^{\prime}(\rho)+\operatorname{div} B \nabla \gamma=g-b . \nabla g .
$$

Now, the theory can be further generalized by taking the deformations of the material into account. These deformations are essentially due to the displacement of atoms in the solid. Thus, it is legitimate to assume that the deformations are infinitesimal and that the displacement gradient is small, in which case we can use the theory of linear elasticity. In that case, the constitutive equation for the chemical potential takes the more general form (see [19])

$$
\mu-b . \nabla \mu=f^{\prime}(\rho)-\alpha \Delta \rho+\beta \frac{\partial \rho}{\partial t}-\mathbf{C}(E-\bar{E}(\rho)) \cdot \bar{E}^{\prime}(\rho)-\gamma
$$

where $\mathbf{C}$ is the elasticity tensor, $E=\frac{1}{2}\left(\nabla u+{ }^{t} \nabla u\right)$ is the linearized deformation tensor, $u$ being the displacement, and $\bar{E}(\rho)$ is the stress free strain at density $\rho$. We assume in this article that $\mathbf{C}$ is a constant symmetric positive definite linear transformation which maps symmetric tensors onto symmetric tensors. Furthermore, as in [19], we consider affine stress free strains of the form $\bar{E}(\rho)=e\left(\rho-\rho_{0}\right) I, e>0, \rho_{0}$ constant, where $I$ is the identity tensor. Proceeding as above, we then obtain the following generalized Cahn-Hilliard equation:

$$
\begin{aligned}
\frac{\partial \rho}{\partial t}-\frac{\partial}{\partial t} b . \nabla \rho-\beta \frac{\partial}{\partial t} \operatorname{div} B \nabla \rho+\alpha \operatorname{div} B \nabla \Delta \rho+ & \frac{e}{2} \operatorname{div} B \nabla \operatorname{Tr}\left[\mathbf{C}\left(\nabla u+{ }^{t} \nabla u\right)\right] \\
& -e^{2} \operatorname{Tr}(\mathbf{C} I) \operatorname{div} B \nabla \rho-\operatorname{div} B \nabla f^{\prime}(\rho)+\operatorname{div} B \nabla \gamma=g-b . \nabla g
\end{aligned}
$$

where Tr denotes the trace operator, which we complete, at first approximation, with the stationary Navier equation of linear elasticity

$$
\operatorname{div} \mathbf{C}\left(\nabla u+{ }^{t} \nabla u\right)-2 e \operatorname{div}(\rho \mathbf{C} I)=0 .
$$


We studied in $[7,8,10,24-27,29]$ some models of generalized Cahn-Hilliard equations under various boundary conditions. However, in all these references, we only considered isotropic materials (i.e. we took $b=0$ ). Our aim in this article is to study more particularly the effects of the term $\frac{\partial}{\partial t} b . \nabla \rho$ from the mathematical point of view.

This article is organized as follows. In Section 2, we obtain some existence and uniqueness results. Then, in Section 3, we study the existence of finite dimensional attractors. First, we give, in Subsection 3.1, a result for a general abstract equation and then apply this result to our models in Subsection 3.2. Finally, we obtain, in Subsection 2.3, the existence of finite dimensional attractors for the complete system (order parameter,displacement) when we take the deformations of the material into account. Part of the results presented in this article is announced in [28].

Throughout this article, the same letter $c$ (and sometimes $c^{\prime}, c^{\prime \prime}$ and $c^{\prime \prime \prime}$ ) shall denote constants which may change from line to line.

\section{EXISTENCE AND UNIQUENESS RESUltS}

\subsection{The main results}

We assume from now on that the medium is represented by the domain $\Omega=\prod_{i=1}^{n}\left(0, L_{i}\right), L_{i}>0, i=$ $1, \ldots, n, n=2$ or 3 , and we assume that all the physical quantities are $\Omega$-periodic. Then, we consider the two following problems:

$$
\begin{gathered}
\frac{\partial \rho}{\partial t}-\frac{\partial}{\partial t} b \cdot \nabla \rho-\beta \frac{\partial}{\partial t} \operatorname{div} B \nabla \rho+ \\
\alpha \operatorname{div} B \nabla \Delta \rho-\operatorname{div} B \nabla f^{\prime}(\rho)+\operatorname{div} B \nabla \gamma=g-b . \nabla g, \\
\rho \text { is } \Omega-\text { periodic ; }
\end{gathered}
$$

and

$$
\begin{aligned}
\frac{\partial \rho}{\partial t}-\frac{\partial}{\partial t} b . \nabla \rho-\beta \frac{\partial}{\partial t} \operatorname{div} B \nabla \rho+\alpha \operatorname{div} B \nabla \Delta \rho+ & \frac{e}{2} \operatorname{div} B \nabla \operatorname{Tr}\left[\mathbf{C}\left(\nabla u+{ }^{t} \nabla u\right)\right] \\
& -e^{2} \operatorname{Tr}(\mathbf{C} I) \operatorname{div} B \nabla \rho-\operatorname{div} B \nabla f^{\prime}(\rho)+\operatorname{div} B \nabla \gamma=g-b . \nabla g, \\
\operatorname{div} \mathbf{C}\left(\nabla u+{ }^{t} \nabla u\right)-2 e \operatorname{div}(\rho \mathbf{C} I)=0, &
\end{aligned}
$$$$
\rho \text { and } u \text { are } \Omega \text { - periodic. }
$$

We assume that $\alpha, \beta$ and $e$ are strictly positive constants, that $b$ is a constant vector, that $B$ is a symmetric positive definite tensor with constant coefficients and that $\mathbf{C}$ is a constant symmetric positive definite linear transformation which maps symmetric tensors onto symmetric tensors. Furthermore, we assume that $f$ is of the form

$$
f(s)=\sum_{i=0}^{2 p+2} a_{i} s^{i}, a_{2 p+2}>0, p \geq 1 .
$$

Finally, we assume that $m(g)=0$, where $m(v)=\frac{1}{V o l(\Omega)} \int_{\Omega} v \mathrm{~d} x$, for $v \in L^{1}(\Omega)$ or $L^{1}(\Omega)^{n}$.

First, we note that if we (formally) integrate (2.1) or (2.3) over $\Omega$, we find

$$
m(\rho)=m(\rho(0)), \forall t \geq 0 .
$$


Furthermore, we note that if $(\rho, u)$ is a solution of $(2.3)-(2.5)$, then so is $(\rho, u+c)$, for every constant vector $c$. Thus, we complete (2.3)-(2.5) with the condition

$$
m(u)=0 ;
$$

we note that we could have taken the condition $m(u)=c$, for every constant vector $c$.

Remark 2.1. We only consider periodic boundary conditions in this article. Neumann boundary conditions are also classical boundary conditions for the Cahn-Hilliard equation. However, for the models introduced above, we would not have in general the conservation of $m(\rho)$ in that case (see [28] for a discussion on the subject).

For the mathematical setting of the problem, we introduce the following spaces:

$$
\begin{gathered}
V_{1}=H_{\mathrm{per}}^{2}(\Omega), \\
V_{2}=\left\{v \in H_{\mathrm{per}}^{1}(\Omega)^{n}, m(v)=0\right\},
\end{gathered}
$$

which we endow with their usual scalar products and norms; in particular, we denote by (.,.) the usual $L^{2}$-scalar product and by $|$.$| the associated norm. Furthermore, for a space W$, we shall denote by $\dot{W}$ the space $\dot{W}=$ $\{v \in W, m(v)=0\}$.

We associate with the above equations the following variational formulations:

Find $\rho:[0, T] \rightarrow V_{1}$ such that

$$
\frac{\mathrm{d}}{\mathrm{d} t}(\rho, q)+\frac{\mathrm{d}}{\mathrm{d} t}(\rho, b . \nabla q)+\beta \frac{\mathrm{d}}{\mathrm{d} t}(B \nabla \rho, \nabla q)+\alpha\left(\nabla B^{\frac{1}{2}} \nabla \rho, \nabla B^{\frac{1}{2}} \nabla q\right)+\left(B \nabla f^{\prime}(\rho), \nabla q\right)=(\varphi, q), \quad \forall q \in V_{1} ;
$$

and find $(\rho, u):[0, T] \rightarrow V_{1} \times V_{2}$ such that

$$
\begin{gathered}
\frac{\mathrm{d} d}{\mathrm{~d} d t}(\rho, q)+\frac{\mathrm{d}}{\mathrm{d} t}(\rho, b . \nabla q)+\beta \frac{\mathrm{d}}{\mathrm{d} t}(B \nabla \rho, \nabla q)+\alpha\left(\nabla B^{\frac{1}{2}} \nabla \rho, \nabla B^{\frac{1}{2}} \nabla q\right)+e^{2} \operatorname{Tr}(\mathbf{C} I)(B \nabla \rho, \nabla q) \\
-\frac{e}{2}\left(B \nabla \operatorname{Tr}\left[\mathbf{C}\left(\nabla u+{ }^{t} \nabla u\right)\right], \nabla q\right)+\left(B \nabla f^{\prime}(\rho), \nabla q\right)=(\varphi, q), \forall q \in V_{1}, \\
\left(\mathbf{C}\left(\nabla u+{ }^{t} \nabla u\right), \nabla \tilde{u}\right)=2 e(\rho(\mathbf{C} I), \nabla \tilde{u}), \forall \tilde{u} \in V_{2}
\end{gathered}
$$

$T>0$, where $\varphi=g-b . \nabla g-\operatorname{div} B \nabla \gamma\left(\right.$ we assume that $\varphi$ belongs to $L^{2}(\Omega)$ ).

We set, for $\rho$ in $V_{1}$ and for $u$ and $\tilde{u}$ in $V_{2}$

$$
a(u, \tilde{u})=\left(\mathbf{C}\left(\nabla u+{ }^{t} \nabla u\right), \nabla \tilde{u}\right), l_{\rho}(\tilde{u})=2 e(\rho(\mathbf{C} I), \nabla \tilde{u})
$$

and we consider the following problem, for $\rho \in L^{2}(\Omega)$ given:

Find $u \in V_{2}$ such that

$$
a(u, \tilde{u})=l_{\rho}(\tilde{u}), \forall \tilde{u} \in V_{2} .
$$

Since $\mathbf{C}$ is symmetric and maps symmetric tensors onto symmetric tensors, we have

$$
a(u, \tilde{u})=\frac{1}{2}\left(\mathbf{C}\left(\nabla u+{ }^{t} \nabla u\right), \nabla \tilde{u}+{ }^{t} \nabla \tilde{u}\right), \forall u, \tilde{u} \in V_{2},
$$

and we then deduce that $a$ is a bilinear selfadjoint coercive and continuous form on $V_{2}$. Furthermore, $l_{\rho}$ is a linear form on $V_{2}$. Thus, we deduce, thanks to Lax-Milgram's theorem, the existence and uniqueness of solutions 
for (2.13). Then, due to classical regularity results on second order elliptic problems, we see that $u$ actually belongs to $\dot{H}_{\mathrm{per}}^{2}(\Omega)^{n}$; more generally, if $\rho \in H_{\mathrm{per}}^{k}(\Omega)$, then $u \in \dot{H}_{\mathrm{per}}^{k+1}(\Omega)^{n}$ and $\|u\|_{H_{\mathrm{per}}^{k+1}(\Omega)^{n}} \leq c(k)\|\rho\|_{H_{\mathrm{per}}^{k}(\Omega)}$.

Now, thanks to (2.13), we can define a mapping

$$
\mathcal{L}: L^{2}(\Omega) \rightarrow \tilde{V}_{2}=\mathcal{L}\left(L^{2}(\Omega)\right) \subset V_{2},
$$

such that $u=\mathcal{L}(\rho)$ is the solution of $(2.13)$ and $\mathcal{L}(\rho) \in \dot{H}_{\text {per }}^{k+1}(\Omega)^{n}$ if $\rho \in H_{\text {per }}^{k}(\Omega)$. We easily prove that $\mathcal{L}$ is linear and Lipschitz. Let us denote by $\overline{\mathcal{L}}$ the restriction of $\mathcal{L}$ to $\dot{L}^{2}(\Omega)$. We can prove, proceeding as in $[7,8]$, that $\overline{\mathcal{L}}$ is invertible and bi-Lipschitz when $\mathbf{C} I$ is positive definite ( $\mathbf{C} I$ is symmetric since $I$ is symmetric); in that case, $\tilde{V}_{2}=\overline{\mathcal{L}}\left(\dot{L}^{2}(\Omega)\right)$ is closed in $V_{2}$. Furthermore, if $u=\mathcal{L}(\rho)$, we have $\rho=m(\rho)+\overline{\mathcal{L}}^{-1}(u)$; and if $(\rho, u)$ is a solution of $(2.10)-(2.11)$, we have (formally) $u(t)=\mathcal{L}(\rho(t))$ and $\rho(t)=m(\rho(0))+\overline{\mathcal{L}}^{-1}(u(t))$, for every $t \geq 0$. Finally, we set

$$
\mathcal{B}(\rho)=-\frac{e}{2} \operatorname{Tr}\left[\mathbf{C}\left(\nabla \mathcal{L}(\rho)+{ }^{t} \nabla \mathcal{L}(\rho)\right)\right]
$$

The mapping $\mathcal{B}$ is linear and satisfies

$$
\|\mathcal{B}(\rho)\|_{H_{\mathrm{per}}^{k}(\Omega)} \leq c(k)\|\rho\|_{H_{\mathrm{per}}^{k}(\Omega)}, \forall \rho \in H_{\mathrm{per}}^{k}(\Omega) .
$$

Thus, we note that (2.10)-(2.11) can be uncoupled in the sense that we first solve the variational problem.

Find $\rho:[0, T] \rightarrow V_{1}$ such that

$$
\begin{aligned}
\frac{\mathrm{d}}{\mathrm{d} t}(\rho, q)+\frac{\mathrm{d}}{\mathrm{d} t}(\rho, b . \nabla q)+\beta \frac{\mathrm{d}}{\mathrm{d} t}(B \nabla \rho, \nabla q)+\alpha\left(\nabla B^{\frac{1}{2}} \nabla \rho, \nabla B^{\frac{1}{2}} \nabla q\right)+e^{2} \operatorname{Tr}(\mathbf{C} I)(B \nabla \rho, \nabla q) \\
+(B \nabla \mathcal{B}(\rho), \nabla q)+\left(B \nabla f^{\prime}(\rho), \nabla q\right)=(\varphi, q), \forall q \in V_{1}
\end{aligned}
$$

and we then set

$$
u(t)=\mathcal{L}(\rho(t)), t \in[0, T] .
$$

We have the following results:

Theorem 2.1. (i) We assume that $\rho_{0} \in H_{\mathrm{per}}^{1}(\Omega) \cap L^{2 p+2}(\Omega)$. Then, (2.9) possesses at least one solution $\rho$ with initial data $\rho_{0}$ satisfying $\rho \in \mathcal{C}\left([0, T] ; H_{\mathrm{per}}^{1}(\Omega) \cap L^{2 p+2}(\Omega)\right) \cap L^{2}\left(0, T ; H_{\mathrm{per}}^{2}(\Omega)\right)$ and $\frac{\partial \rho}{\partial t} \in L^{2}\left(0, T ; L^{2}(\Omega)\right), \forall T>0$. Furthermore, if $p=1$ or 2 when $n=3$ ( $p$ is arbitrary when $n=2$ ), then this solution is unique.

(ii) If furthermore $\rho_{0} \in H_{\mathrm{per}}^{2}(\Omega)$ and $p=1$ or 2 when $n=3$, then $\rho \in \mathcal{C}\left([0, T] ; H_{\text {per }}^{2}(\Omega)\right)$ and $\frac{\partial \rho}{\partial t} \in$ $L^{2}\left(0, T ; H_{\mathrm{per}}^{1}(\Omega)\right)$.

Theorem 2.2. (i) We assume that $\rho_{0} \in H_{\mathrm{per}}^{1}(\Omega) \cap L^{2 p+2}(\Omega)$. Then, (2.16)-(2.17) possesses at least one solution $(\rho, u)$ with initial data for the order parameter $\rho_{0}$ satisfying $\rho \in \mathcal{C}\left([0, T] ; H_{\mathrm{per}}^{1}(\Omega) \cap L^{2 p+2}(\Omega)\right) \cap L^{2}\left(0, T ; H_{\mathrm{per}}^{2}(\Omega)\right)$, $\frac{\partial \rho}{\partial t} \in L^{2}\left(0, T ; L^{2}(\Omega)\right)$ and $u \in \mathcal{C}\left([0, T] ; H_{\mathrm{per}}^{2}(\Omega)^{n}\right) \cap L^{2}\left(0, T ; H_{\mathrm{per}}^{3}(\Omega)^{n}\right), \forall T>0$. Furthermore, if $p=1$ or 2 when $n=3$, then this solution is unique.

(ii) If furthermore $\rho_{0} \in H_{\mathrm{per}}^{2}(\Omega)$ and $p=1$ or 2 when $n=3$, then $\rho \in \mathcal{C}\left([0, T] ; H_{\mathrm{per}}^{2}(\Omega)\right)$, $\frac{\partial \rho}{\partial t} \in L^{2}\left(0, T ; H_{\mathrm{per}}^{1}(\Omega)\right)$ and $u \in \mathcal{C}\left([0, T] ; H_{\text {per }}^{3}(\Omega)^{n}\right)$.

\subsection{Proof of Theorems 2.1 and 2.2}

First, we have the following lemma :

Lemma 2.1. The map $\rho \mapsto\left|\nabla B^{\frac{1}{2}} \nabla \rho\right|+|\rho|$ defines a norm on $V_{1}$ that is equivalent to the usual $H^{2}-$ norm. 
Proof. First, we note that thanks to classical regularity results on second order elliptic problems (see [1] and [4]), $\left|\operatorname{div} B^{\frac{1}{2}} \nabla.\right|+|$.$| is a norm on V_{1}$ that is equivalent to the usual $H^{2}-$ norm. Then, we have, for $\rho \in V_{1}$

$$
\left|\nabla B^{\frac{1}{2}} \nabla \rho\right|^{2}=\sum_{i, j=1}^{n}\left|\frac{\partial}{\partial x_{i}}\left(B^{\frac{1}{2}} \nabla \rho\right)_{j}\right|^{2} \geq \sum_{i=1}^{n}\left|\frac{\partial}{\partial x_{i}}\left(B^{\frac{1}{2}} \nabla \rho\right)_{i}\right|^{2},
$$

where $u_{i}$ denotes the $i$-th component of the vector $u$. Thus, if $\epsilon>0$ is small enough, we find

$$
\left|\nabla B^{\frac{1}{2}} \nabla \rho\right|^{2} \geq \epsilon\left|\operatorname{div} B^{\frac{1}{2}} \nabla \rho\right|^{2}
$$

hence the result.

Now, we turn to the proof of Theorems 2.1 and 2.2.

We note that it is sufficient here to study the variational formulation (2.16). Indeed, (2.9) is a particular case of (2.16) corresponding to $e=0$. All the estimates that we shall derive below will be valid for both problems.

We shall only derive formal a priori estimates here. These estimates, and thus the existence results, can be justified by making Galerkin approximations. Furthermore, the passage to the limit in the nonlinear term will be based on classical Aubin-Lions compactness results (see [20]); indeed, we note that we shall easily derive the proper a priori estimates on $\frac{\partial \rho}{\partial t}$.

We take $q=N^{-1} \bar{\rho}$ in (2.16), where $\bar{\rho}=\rho-m(\rho)$, and obtain, integrating by parts and setting $\|\cdot\|_{-1}=\mid N^{-\frac{1}{2}}$.

$$
\begin{aligned}
\frac{1}{2} \frac{\mathrm{d}}{\mathrm{d} t}\|\bar{\rho}\|_{-1}^{2}+\frac{\beta}{2} \frac{\mathrm{d}}{\mathrm{d} t}|\rho|^{2}+\alpha|\nabla \rho|^{2}+e^{2} \operatorname{Tr}(\mathbf{C} I)|\bar{\rho}|^{2}+\left(f^{\prime}(\rho), \rho\right) & =\left(\varphi, N^{-1} \bar{\rho}\right) \\
+ & \left(f^{\prime}(\rho), m\left(\rho_{0}\right)\right)-\left(\frac{\partial \rho}{\partial t}, b . \nabla N^{-1} \bar{\rho}\right)-(\mathcal{B}(\rho), \bar{\rho}) .
\end{aligned}
$$

We have, using (2.15) with $k=0$

$$
|(\mathcal{B}(\rho), \bar{\rho})| \leq c(0)|\rho||\bar{\rho}| \leq c|\rho|^{2},
$$

and we obtain, noting that $\left(f^{\prime}(\rho), \rho\right) \geq(p+1) a_{2 p+2} \int_{\Omega} \rho^{2 p+2} \mathrm{~d} x-c, c \geq 0$, an inequality of the form

$$
\frac{\mathrm{d}}{\mathrm{d} t}\left(\|\bar{\rho}\|_{-1}^{2}+\beta|\rho|^{2}\right)+\alpha|\nabla \rho|^{2}+e^{2} \operatorname{Tr}(\mathbf{C} I)|\bar{\rho}|^{2}+2(p+1) a_{2 p+2} \int_{\Omega} \rho^{2 p+2} \mathrm{~d} x \leq c|\rho|^{2}-2\left(b . \nabla \rho, N^{-1} \frac{\partial \rho}{\partial t}\right)+c^{\prime} .
$$

Then, we take $q=N^{-1} \frac{\partial \rho}{\partial t}$ in $(2.16)$ and find

$$
\begin{aligned}
\frac{\alpha}{2} \frac{\mathrm{d}}{\mathrm{d} t}|\nabla \rho|^{2}+\frac{\mathrm{d}}{\mathrm{d} t} \int_{\Omega} f(\rho) \mathrm{d} x+\frac{1}{2} e^{2} \operatorname{Tr}(\mathbf{C} I) \frac{\mathrm{d}}{\mathrm{d} t}|\rho|^{2}+\left\|\frac{\partial \rho}{\partial t}\right\|_{-1}^{2}+\beta\left|\frac{\partial \rho}{\partial t}\right|^{2} & =\left(\varphi, N^{-1} \frac{\partial \rho}{\partial t}\right) \\
- & \left(\frac{\partial \rho}{\partial t}, b . \nabla N^{-1} \frac{\partial \rho}{\partial t}\right)-\left(\mathcal{B}(\rho), N^{-1} \frac{\partial \rho}{\partial t}\right) .
\end{aligned}
$$

Since $b . \nabla$ and $N$ commute, we deduce that $b . \nabla$ and $N^{-1}$ commute. Therefore, $b . \nabla N^{-1}$ is antisymmetric on $\dot{L}^{2}(\Omega)$ and we have

$$
\left(\frac{\partial \rho}{\partial t}, b \cdot \nabla N^{-1} \frac{\partial \rho}{\partial t}\right)=0
$$

in (2.23). Then, we obtain, thanks to (2.23) and (2.24) (and also (2.15))

$$
\frac{\mathrm{d}}{\mathrm{d} t}\left(\alpha|\nabla \rho|^{2}+e^{2} \operatorname{Tr}(\mathbf{C} I)|\rho|^{2}+2 \int_{\Omega} f(\rho) \mathrm{d} x\right)+\left\|\frac{\partial \rho}{\partial t}\right\|_{-1}^{2}+\beta\left|\frac{\partial \rho}{\partial t}\right|^{2} \leq c|\rho|^{2}+c^{\prime}
$$


We write, in $(2.22)$

$$
\left|2\left(b . \nabla \rho, N^{-1} \frac{\partial \rho}{\partial t}\right)\right|=\left|2\left(\rho, b . \nabla N^{-1} \frac{\partial \rho}{\partial t}\right)\right| \leq c(\zeta)\left\|\frac{\partial \rho}{\partial t}\right\|_{-1}^{2}+\zeta|\rho|^{2} \leq c\left|\frac{\partial \rho}{\partial t}\right|^{2}+(p+1) a_{2 p+2} \int_{\Omega} \rho^{2 p+2} \mathrm{~d} x+c^{\prime}
$$

choosing $\zeta>0$ properly, and we find, summing (2.25) and $\epsilon(2.22)$, where $\epsilon>0$ is small enough, an inequality of the form

$$
\begin{aligned}
\frac{\mathrm{d}}{\mathrm{d} t}\left(\epsilon\|\bar{\rho}\|_{-1}^{2}+\epsilon \beta|\rho|^{2}+\alpha|\nabla \rho|^{2}+e^{2} \operatorname{Tr}(\mathbf{C} I)|\rho|^{2}+2 \int_{\Omega} f(\rho) \mathrm{d} x\right)+\epsilon\left(\alpha|\nabla \rho|^{2}+e^{2} \operatorname{Tr}(\mathbf{C} I)|\bar{\rho}|^{2}\right) & +c\left|\frac{\partial \rho}{\partial t}\right|^{2}+c^{\prime} \int_{\Omega} \rho^{2 p+2} \mathrm{~d} x \leq c^{\prime \prime}|\rho|^{2}+c^{\prime \prime \prime}
\end{aligned}
$$

We set

$$
E(t)=\epsilon\|\bar{\rho}\|_{-1}^{2}+\epsilon \beta|\rho|^{2}+\alpha|\nabla \rho|^{2}+e^{2} \operatorname{Tr}(\mathbf{C} I)|\rho|^{2}+2 \int_{\Omega} f(\rho) \mathrm{d} x
$$

Since

$$
\frac{a_{2 p+2}}{2} \int_{\Omega} \rho^{2 p+2} \mathrm{~d} x-c_{0} \leq \int_{\Omega} f(\rho) \mathrm{d} x
$$

where $c_{0} \geq 0$, we deduce from (2.27) an inequality of the form

$$
\frac{\mathrm{d} E}{\mathrm{~d} t}+c\left|\frac{\partial \rho}{\partial t}\right|^{2} \leq c^{\prime} E+c^{\prime \prime}
$$

Indeed, we write, considering the right-hand-side of (2.27):

$$
c^{\prime \prime}|\rho|^{2} \leq c^{\prime \prime \prime}\left(\epsilon\|\bar{\rho}\|_{-1}^{2}+\epsilon \beta|\rho|^{2}+\alpha|\nabla \rho|^{2}+e^{2} \operatorname{Tr}(\mathbf{C} I)|\rho|^{2}+a_{2 p+2} \int_{\Omega} \rho^{2 p+2} \mathrm{~d} x\right) \leq c^{\prime \prime \prime} E(t)+2 c_{0} c^{\prime \prime \prime} .
$$

Now, we take $q=\rho$ in (2.16) and have

$$
\begin{aligned}
\frac{1}{2} \frac{\mathrm{d}}{\mathrm{d} t}|\rho|^{2}+\frac{\beta}{2} \frac{\mathrm{d}}{\mathrm{d} t}(B \nabla \rho, \nabla \rho)+\alpha\left|\nabla B^{\frac{1}{2}} \nabla \rho\right|^{2}+e^{2} \operatorname{Tr}(\mathbf{C} I)(B \nabla \rho, \nabla \rho) & +\left(B \nabla f^{\prime}(\rho), \nabla \rho\right) \\
& =(\varphi, \rho)-\left(\frac{\partial \rho}{\partial t}, b . \nabla \rho\right)-(B \nabla \mathcal{B}(\rho), \nabla \rho) .
\end{aligned}
$$

We deduce from $(2.15)$ (with $k=1$ ), (2.31) and the inequality

$$
\left(B \nabla f^{\prime}(\rho), \nabla \rho\right)=\left(f^{\prime \prime}(\rho) B \nabla \rho, \nabla \rho\right) \geq \frac{a_{2 p+2}}{2} \int_{\Omega} \rho^{2 p}|\nabla \rho|^{2} \mathrm{~d} x-c(B \nabla \rho, \nabla \rho), c \geq 0,
$$

that

$$
\frac{\mathrm{d}}{\mathrm{d} t}\left(|\rho|^{2}+\beta(B \nabla \rho, \nabla \rho)\right)+\alpha\left|\nabla B^{\frac{1}{2}} \nabla \rho\right|^{2}+e^{2} \operatorname{Tr}(\mathbf{C} I)(B \nabla \rho, \nabla \rho)+c \int_{\Omega} \rho^{2 p}|\nabla \rho|^{2} \mathrm{~d} x \leq c^{\prime}\|\rho\|_{H_{\mathrm{per}}^{1}}^{2}(\Omega)+c^{\prime \prime}\left|\frac{\partial \rho}{\partial t}\right|^{2}+c^{\prime \prime \prime} .
$$


Finally, we take $q=\frac{\partial \rho}{\partial t}$ in $(2.16)$ and obtain

$$
\begin{aligned}
\frac{\alpha}{2} \frac{\mathrm{d}}{\mathrm{d} t}\left|\nabla B^{\frac{1}{2}} \nabla \rho\right|^{2}+\frac{1}{2} e^{2} \operatorname{Tr}(\mathbf{C} I) \frac{\mathrm{d}}{\mathrm{d} t}(B \nabla \rho, \nabla \rho)+\left|\frac{\partial \rho}{\partial t}\right|^{2}+\beta & \left(B \nabla \frac{\partial \rho}{\partial t}, \nabla \frac{\partial \rho}{\partial t}\right)+\left(B \nabla f^{\prime}(\rho), \nabla \frac{\partial \rho}{\partial t}\right) \\
& =\left(g, \frac{\partial \rho}{\partial t}\right)-\left(B \nabla \mathcal{B}(\rho), \nabla \frac{\partial \rho}{\partial t}\right)-\left(b . \nabla \frac{\partial \rho}{\partial t}, \frac{\partial \rho}{\partial t}\right) .
\end{aligned}
$$

We assume that $p=1$ or 2 when $n=3$. When $n=2$, we write

$$
\begin{aligned}
\left|\left(B \nabla f^{\prime}(\rho), \nabla \frac{\partial \rho}{\partial t}\right)\right| & \leq c \int_{\Omega}\left|f^{\prime \prime}(\rho)\right||\nabla \rho|\left|\nabla \frac{\partial \rho}{\partial t}\right| \mathrm{d} x \\
& \leq c\left|\nabla \frac{\partial \rho}{\partial t}\right|\left\|f^{\prime \prime}(\rho)\right\|_{L^{4}(\Omega)}\|\nabla \rho\|_{L^{4}(\Omega)^{n}} \\
& \leq c\left|\nabla \frac{\partial \rho}{\partial t}\right|\left(\|\rho\|_{H_{\mathrm{per}}^{1}(\Omega)}^{2 p}+1\right)\|\rho\|_{H_{\mathrm{per}}^{2}(\Omega)} .
\end{aligned}
$$

When $n=3$, we consider the term $\int_{\Omega}|\rho|^{4}|\nabla \rho|\left|\nabla \frac{\partial \rho}{\partial t}\right| \mathrm{d} x$, the other terms being easier to treat. We have, using Agmon's inequality $\|\rho\|_{L^{\infty}(\Omega)} \leq c\|\rho\|_{H_{\mathrm{per}}^{1}(\Omega)}^{\frac{1}{2}}\|\rho\|_{H_{\mathrm{per}}^{2}(\Omega)}^{\frac{1}{2}}, \forall \rho \in H_{\mathrm{per}}^{2}(\Omega)$ (see for instance [31])

$$
\int_{\Omega}|\rho|^{4}|\nabla \rho|\left|\nabla \frac{\partial \rho}{\partial t}\right| \mathrm{d} x \leq\|\rho\|_{L^{\infty}(\Omega)}^{2}\|\rho\|_{L^{6}(\Omega)}^{2}\|\nabla \rho\|_{L^{6}(\Omega)^{n}}\left|\nabla \frac{\partial \rho}{\partial t}\right| \leq c\|\rho\|_{H_{\mathrm{per}}^{1}(\Omega)}^{3}\|\rho\|_{H_{\mathrm{per}}^{2}(\Omega)}^{2}\left|\nabla \frac{\partial \rho}{\partial t}\right| .
$$

In both cases, we have an inequality of the form

$$
\left|\left(B \nabla f^{\prime}(\rho), \nabla \frac{\partial \rho}{\partial t}\right)\right| \leq \zeta\left(B \nabla \frac{\partial \rho}{\partial t}, \nabla \frac{\partial \rho}{\partial t}\right)+c\left(\|\rho\|_{H_{\mathrm{per}}^{1}(\Omega)}^{2}+1\right)^{r}\left(\|\rho\|_{H_{\mathrm{per}}^{2}(\Omega)}^{2}+1\right)\|\rho\|_{H_{\mathrm{per}}^{2}(\Omega)}^{2},
$$

for every $\zeta>0$, where $r$ is a nonnegative integer. Then, we deduce from (2.33), using (2.15) and noting that $b . \nabla$ is antisymmetric on $H_{\mathrm{per}}^{1}(\Omega)$

$$
\begin{aligned}
\frac{\mathrm{d}}{\mathrm{d} t}\left(\alpha\left|\nabla B^{\frac{1}{2}} \nabla \rho\right|^{2}+e^{2} \operatorname{Tr}(\mathbf{C} I)(B \nabla \rho, \nabla \rho)\right)+\left|\frac{\partial \rho}{\partial t}\right|^{2}+ & \beta\left(B \nabla \frac{\partial \rho}{\partial t}, \nabla \frac{\partial \rho}{\partial t}\right) \leq c\|\rho\|_{H_{\mathrm{per}}^{1}(\Omega)}^{2} \\
& +c^{\prime}\left(\|\rho\|_{H_{\mathrm{per}}^{1}(\Omega)}^{2}+1\right)^{r}\left(\|\rho\|_{H_{\mathrm{per}}^{2}(\Omega)}^{2}+1\right)\|\rho\|_{H_{\mathrm{per}}^{2}(\Omega)}^{2}+c^{\prime \prime}
\end{aligned}
$$

The existence results in Theorems 2.1 and 2.2 are then based on $(2.30,2.32,2.37)$ and Lemma 2.1.

Now, we consider two solutions $\rho_{1}$ and $\rho_{2}$ of (2.16) with the same initial data. Setting $\rho=\rho_{1}-\rho_{2}$, we find, proceeding as above

$$
\frac{\mathrm{d} \bar{E}}{\mathrm{~d} t}+c\left|\frac{\partial \rho}{\partial t}\right|^{2}+c^{\prime}\left(f^{\prime}\left(\rho_{1}\right)-f^{\prime}\left(\rho_{2}\right), \rho\right)+c^{\prime \prime}\left(f^{\prime}\left(\rho_{1}\right)-f^{\prime}\left(\rho_{2}\right), \frac{\partial \rho}{\partial t}\right) \leq c^{\prime \prime \prime}|\rho|^{2},
$$

$c, c^{\prime}, c^{\prime \prime}>0$, where

$$
\bar{E}(t)=\epsilon|| \bar{\rho} \|_{-1}^{2}+\epsilon \beta|\rho|^{2}+\alpha|\nabla \rho|^{2}+e^{2} \operatorname{Tr}(\mathbf{C} I)|\rho|^{2},
$$

with $\epsilon$ as above. We have

$$
\left(f^{\prime}\left(\rho_{1}\right)-f^{\prime}\left(\rho_{2}\right), \rho\right) \geq-c|\rho|^{2}, c \geq 0
$$


and $\left(f^{\prime}\left(\rho_{1}\right)-f^{\prime}\left(\rho_{2}\right), \frac{\partial \rho}{\partial t}\right)=\int_{\Omega} h\left(\rho_{1}, \rho_{2}\right) \rho \frac{\partial \rho}{\partial t} \mathrm{~d} x$, where $h$ is of degree $2 p$ with respect to $\rho_{1}$ and $\rho_{2}$. Then, we write, for $n=2$

$$
\begin{aligned}
\left|\left(f^{\prime}\left(\rho_{1}\right)-f^{\prime}\left(\rho_{2}\right), \frac{\partial \rho}{\partial t}\right)\right| & \leq c\left(\left\|\rho_{1}\right\|_{L^{6 p}(\Omega)}^{2 p}+\left\|\rho_{2}\right\|_{L^{6 p}(\Omega)}^{2 p}+1\right)\|\rho\|_{L^{6}(\Omega)}\left|\frac{\partial \rho}{\partial t}\right| \\
& \leq c\left(\left\|\rho_{1}\right\|_{H_{\mathrm{per}}^{1}(\Omega)}^{2 p}+\left\|\rho_{2}\right\|_{H_{\mathrm{per}}^{1}(\Omega)}^{2 p}+1\right)\|\rho\|_{H_{\mathrm{per}}^{1}(\Omega)}\left|\frac{\partial \rho}{\partial t}\right| .
\end{aligned}
$$

When $n=3$, we use Agmon's inequality as in (2.35) and find, recalling that $p=1$ or 2

$$
\begin{aligned}
\left|\left(f^{\prime}\left(\rho_{1}\right)-f^{\prime}\left(\rho_{2}\right), \frac{\partial \rho}{\partial t}\right)\right| & \leq c\left(\left\|\rho_{1}\right\|_{H_{\mathrm{per}}^{1}(\Omega)}^{3}+\left\|\rho_{2}\right\|_{H_{\mathrm{per}}^{1}(\Omega)}^{3}+1\right)\left(\left\|\rho_{1}\right\|_{H_{\mathrm{per}}^{2}(\Omega)}+\left\|\rho_{2}\right\|_{H_{\mathrm{per}}^{2}(\Omega)}+1\right) \\
& \times\|\rho\|_{H_{\mathrm{per}}^{1}(\Omega)}\left|\frac{\partial \rho}{\partial t}\right| .
\end{aligned}
$$

Finally, we obtain, in both cases, an inequality of the form

$$
\frac{\mathrm{d} \bar{E}}{\mathrm{~d} t} \leq c\left(\left\|\rho_{1}\right\|_{H_{\mathrm{per}}^{1}(\Omega)}^{2}+\left\|\rho_{2}\right\|_{H_{\mathrm{per}}^{1}(\Omega)}^{2}+1\right)^{r}\left(\left\|\rho_{1}\right\|_{H_{\mathrm{per}}^{2}(\Omega)}^{2}+\left\|\rho_{2}\right\|_{H_{\mathrm{per}}^{2}(\Omega)}^{2}+1\right) \bar{E},
$$

$r \in N^{\star}$, hence the uniqueness using Gronwall's Lemma.

\section{Existence of Finite Dimensional ATtRACTORS}

\subsection{An abstract result}

We consider the following equation in a Hilbert space $H$ endowed with the scalar product (...) and associated norm |.|:

$$
\begin{gathered}
\frac{\mathrm{d} L u}{\mathrm{~d} t}+A u+\mathcal{R}(u)=0, \\
u(0)=u_{0} .
\end{gathered}
$$

We assume that $A$ is a linear selfadjoint strictly positive operator with compact inverse and that $L=L_{1}+L_{2}$, where $L_{1}$ is a linear selfadjoint strictly positive operator with compact inverse and where $L_{2}$ is a linear antisymmetric operator. Furthermore, we assume that there exists $r \in\left(0, \frac{1}{2}\right)$ such that $D\left(L_{1}\right)=D\left(A^{2 r}\right)$. We endow the space $D\left(A^{s}\right)$ with the norm $\mid A^{s}$. $\mid$ and we assume that the norms $\left|L_{1}^{\frac{s}{2 r}}.\right|$ and $\left|A^{s}.\right|$ are equivalent on $D\left(A^{s}\right), \forall s \in R$.

We assume that (3.1)-(3.2) is well posed and that we can define the continuous semigroup

$$
\begin{aligned}
S(t): K \subset D\left(A^{r}\right) & \rightarrow K \\
u_{0} & \mapsto u(t),
\end{aligned}
$$

$t \geq 0$, where $K$ is a closed subset of $D\left(A^{r}\right)$ and $u$ is the solution of (3.1)-(3.2). Then, we assume that $\mathcal{R}: K \cap D\left(A^{d}\right) \rightarrow H, d \geq r$, and that $S(t)\left(K \cap D\left(A^{d}\right)\right) \subset K \cap D\left(A^{d}\right), \forall t \geq 0$. Finally, we assume that the semigroup $S(t)$ possesses the global attractor $\mathcal{A}_{K}$ on $K$.

We consider here situations in which $K$ is not necessarily a vector nor an affine space so that the usual method, based on the Lyapunov exponents, which gives the finite dimensionality of the global attractor for a semigroup (see for instance [31]) cannot be applied. We shall instead prove the existence of exponential attractors (which, by definition, contain the global attractor and have finite fractal dimension, see [13]). To 
do so, we need to prove the so-called squeezing property (see [13]). A first method, which gives the squeezing property, is described in [13]. This method is based on an inequality satisfied by a quotient of norms. Because of the operator $L_{2}$, this method cannot be used here (when $L_{2} \neq 0$ ). We shall instead use an idea of [2] based on a decomposition of the difference of two trajectories. This second method, although it gives less sharp estimates on the fractal dimension of the exponential attractors that we obtain (but this is not an important issue in this article), is actually more general than that of [13]. Indeed, it can generally be applied in the situations in which the method of [13] can be used, but it has also been applied with success in many situations in which the method of [13] could not be used (see [2,14,17,18,24-27]).

We have the following result (see [24]):

Proposition 3.1. Let $E$ and $V$ be two Hilbert spaces such that the inclusion $V \subset E$ is compact and let $S(t)$ be a semigroup acting on $K$, where $K$ is a closed subset of $E$. Furthermore, let us assume that there exist orthogonal projectors $P_{n}: E \rightarrow E$ with finite rank $n$ such that $\left|Q_{n} y\right|_{E} \leq c(n)|y|_{V}, \forall y \in V$, where $Q_{n}=I-P_{n}$ and $c(n) \rightarrow 0$ as $n \rightarrow+\infty$. Let $X$ be a closed positively invariant subset of $K$. If $S(t) \varphi-S(t) \psi=\varphi^{1}(t)+\varphi^{2}(t)$, $\left|\varphi^{1}(t)\right|_{E} \leq d(t)|\varphi-\psi|_{E},\left|\varphi^{2}(t)\right|_{V} \leq h(t)|\varphi-\psi|_{E}, \forall \varphi, \psi \in X$, where $d(t)$ is continuous and satisfies $d(t) \rightarrow 0$ as $t \rightarrow+\infty$ and $h(t)$ is continuous, then $S(t)$ enjoys the squeezing property on $X$.

In order to apply this result to the semigroup $S(t)$ associated with (3.1)-(3.2), we set $E=D\left(L_{1}^{\frac{1}{2}}\right), V=D\left(L_{1}^{\frac{1}{4 r}}\right)$ and $P_{n}$ the orthogonal projector on the $n$ first eigenvalues of $L_{1}$. Then, we assume that there exists a closed set $X \subset K$ that is positively invariant by $S(t)$.

Let $u_{0}$ and $v_{0}$ belong to $X$. We consider the following decomposition:

$$
S(t) u_{0}-S(t) v_{0}=w^{1}+w^{2},
$$

where $w^{1}$ and $w^{2}$ are the solutions of

$$
\begin{gathered}
\frac{\mathrm{d} L_{1} w^{1}}{\mathrm{~d} t}+A w^{1}=0 \\
w^{1}(0)=w_{0}
\end{gathered}
$$

and

$$
\begin{gathered}
\frac{\mathrm{d} L w^{2}}{\mathrm{~d} t}+A w^{2}+\mathcal{R}(u)-\mathcal{R}(v)+\frac{\mathrm{d} L_{2} w^{1}}{\mathrm{~d} t}=0, \\
w^{2}(0)=0
\end{gathered}
$$

respectively.

We assume that $\forall u_{0}, v_{0} \in X$, then $w^{1}(t) \in D\left(A^{\frac{1}{2}}\right), w^{2}(t) \in D\left(A^{\frac{1}{2}}\right)$ and $\frac{\mathrm{d} w^{2}}{\mathrm{~d} t}(t) \in D\left(L_{1}^{\frac{1}{2}}\right)$ (at least a.e. $t$ ) and that

$$
|\mathcal{R}(u)-\mathcal{R}(v)|^{2} \leq k_{1}(t)\left|A^{\frac{1}{2}} w^{2}\right|^{2}+k_{2}(t)\left|L_{1}^{\frac{1}{2}} w^{1}\right|^{2}+k_{3}\left|A^{\frac{1}{2}} w^{1}\right|^{2}
$$

or

$$
\left|\left(\mathcal{R}(u)-\mathcal{R}(v), \frac{\mathrm{d} w^{2}}{\mathrm{~d} t}\right)\right| \leq k_{1}(t)\left|A^{\frac{1}{2}} w^{2}\right|^{2}+k_{2}(t)\left|L_{1}^{\frac{1}{2}} w^{1}\right|^{2}+k_{3}\left|A^{\frac{1}{2}} w^{1}\right|^{2}+\frac{1}{2}\left|L_{1}^{\frac{1}{2}} \frac{\mathrm{d} w^{2}}{\mathrm{~d} t}\right|^{2},
$$

where $k_{1}$ and $k_{2}$ are continuous and $k_{3}$ is a constant (and $k_{1}, k_{2}$ and $k_{3}$ only depend on $X$ ). 
Now, we are going to derive formal a priori estimates. Again, these estimates can be justified by making proper Galerkin approximations (see [24] for more details).

We take the scalar product in $H$ of (3.4) with $w^{1}$ and obtain

$$
\frac{1}{2} \frac{\mathrm{d}}{\mathrm{d} t}\left|L_{1}^{\frac{1}{2}} w^{1}\right|^{2}+\left|A^{\frac{1}{2}} w^{1}\right|^{2}=0
$$

which yields

$$
\frac{\mathrm{d}}{\mathrm{d} t}\left|L_{1}^{\frac{1}{2}} w^{1}\right|^{2}+c\left|L_{1}^{\frac{1}{2}} w^{1}\right|^{2} \leq 0
$$

and thus

$$
\left|L_{1}^{\frac{1}{2}} w^{1}\right| \leq e^{-c t}\left|L_{1}^{\frac{1}{2}} w_{0}\right|, c>0 .
$$

Furthermore, we deduce from (3.10) that

$$
\int_{0}^{t}\left|A^{\frac{1}{2}} w^{1}\right|^{2} \mathrm{~d} \tau \leq \frac{1}{2}\left|L_{1}^{\frac{1}{2}} w_{0}\right|^{2}
$$

Now, we take the scalar product in $H$ of (3.6) with $\frac{\mathrm{d} w^{2}}{\mathrm{~d} t}$ and have

$$
\frac{1}{2} \frac{\mathrm{d}}{\mathrm{d} t}\left|A^{\frac{1}{2}} w^{2}\right|^{2}+\left|L_{1}^{\frac{1}{2}} \frac{\mathrm{d} w^{2}}{\mathrm{~d} t}\right|^{2}+\left(L_{2} \frac{\mathrm{d} w^{2}}{\mathrm{~d} t}, \frac{\mathrm{d} w^{2}}{\mathrm{~d} t}\right)+\left(\mathcal{R}(u)-\mathcal{R}(v), \frac{\mathrm{d} w^{2}}{\mathrm{~d} t}\right)+\left(\frac{\mathrm{d} L_{2} w^{1}}{\mathrm{~d} t}, \frac{\mathrm{d} w^{2}}{\mathrm{~d} t}\right)=0 .
$$

We deduce from (3.4) that $\frac{d w^{1}}{d t}=-L_{1}^{-1} A w^{1}$, which yields

$$
\frac{\mathrm{d} L_{2} w^{1}}{\mathrm{~d} t}=-L_{2} L_{1}^{-1} A w^{1} .
$$

Therefore

$$
\left(\frac{\mathrm{d} L_{2} w^{1}}{\mathrm{~d} t}, \frac{\mathrm{d} w^{2}}{\mathrm{~d} t}\right)=-\left(L_{2} L_{1}^{-1} A w^{1}, \frac{\mathrm{d} w^{2}}{\mathrm{~d} t}\right)=\left(L_{1}^{-1} A w^{1}, L_{2} \frac{\mathrm{d} w^{2}}{\mathrm{~d} t}\right)
$$

Assuming that

$$
\left|L_{1}^{-1} A u\right| \leq c\left|A^{\frac{1}{2}} u\right|, \forall u \in D\left(A^{\frac{1}{2}}\right),
$$

and that

$$
\left|L_{2} u\right| \leq c\left|L_{1}^{\frac{1}{2}} u\right|, \forall u \in D\left(L_{1}^{\frac{1}{2}}\right)
$$

we deduce that

$$
\left|\left(\frac{\mathrm{d} L_{2} w^{1}}{\mathrm{~d} t}, \frac{\mathrm{d} w^{2}}{\mathrm{~d} t}\right)\right| \leq \frac{1}{2}\left|L_{1}^{\frac{1}{2}} \frac{\mathrm{d} w^{2}}{\mathrm{~d} t}\right|^{2}+c\left|A^{\frac{1}{2}} w^{1}\right|^{2}
$$

Thus, we deduce from (3.13), using (3.8) (or (3.9)), that

$$
\frac{1}{2} \frac{\mathrm{d}}{\mathrm{d} t}\left|A^{\frac{1}{2}} w^{2}\right|^{2} \leq k_{1}(t)\left|A^{\frac{1}{2}} w^{2}\right|^{2}+k_{2}(t)\left|L_{1}^{\frac{1}{2}} w^{1}\right|^{2}+k_{3}^{\prime}\left|A^{\frac{1}{2}} w^{1}\right|^{2},
$$


where $k_{3}^{\prime}=k_{3}+$ Constant. Then, we deduce from (3.19), using Gronwall's Lemma, (3.11) and (3.12), that

$$
\left|A^{\frac{1}{2}} w^{2}\right|^{2} \leq h(t)\left|L_{1}^{\frac{1}{2}} w_{0}\right|^{2}
$$

where $h(t)=2\left[\int_{0}^{t} e^{-c s} k_{2}(s) e^{2 \int_{s}^{t} k_{1}(\tau) \mathrm{d} \tau} \mathrm{d} s+k_{3}^{\prime} e^{2 \int_{s}^{t} k_{1}(\tau) \mathrm{d} \tau}\right]$, which yields

$$
\left|L_{1}^{\frac{1}{4 r}} w^{2}\right| \leq c \sqrt{h(t)}\left|L_{1}^{\frac{1}{2}} w_{0}\right|
$$

Thus, we deduce that $S(t)$ enjoys the squeezing property on $X$ for the topology of $D\left(L_{1}^{\frac{1}{2}}\right)$. Now, if $S(t)$ is Lipschitz on $X$ and if $X$ can be covered by a finite number of balls of radius one if it is not compact, see [2], then $S(t)$ possesses an exponential attractor $\mathcal{M}_{K}$ on $X$. We deduce that $\mathcal{A}_{K}$ (which is also the global attractor for $S(t)$ on $X$ in general) has finite fractal dimension.

Remark 3.1. (i) We can easily adapt the proofs presented above to the nonautonomous (quasiperiodic) case (i.e. when $\mathcal{R}$ depends explicitly and quasi periodically on the time). We refer the interested reader to $[9,16,18$, $23,26]$ for more details.

(ii) We also have a similar result when $L_{2}=0$. In that case however, we derived in [24] and [26] similar estimates by taking the scalar product in $H$ of (3.6) with $L w^{2}$. This estimate allows us to use the regularizing effects of the operator $L$ and, in some situations, it necessitates less regularity (typically when $r \in\left(0, \frac{1}{4}\right)$; this will not be the case for the equations that we study in this article, for which we shall have $r=\frac{1}{4}$ ). When $L_{2} \neq 0$, we can also use this estimate, but we would need to have $L$ positive and to have the norms $\mid L_{1}$. $\mid$ and $\mid L$. equivalent on $D\left(L_{1}\right)$. The problem, for the models considered in this article, is that this would require to have $b$ small, which is too restrictive.

\subsection{Application to the generalized Cahn-Hilliard equations}

Thanks to the results obtained in Section 2, we can define, for $p=1$ or 2 when $n=3$, the continuous semigroup

$$
\begin{aligned}
S(t): H_{\mathrm{per}}^{1}(\Omega) & \rightarrow H_{\mathrm{per}}^{1}(\Omega) \\
\rho_{0} & \mapsto \rho(t),
\end{aligned}
$$

$t \geq 0$, where $\rho$ is the solution of (2.16) with initial data $\rho_{0}$.

First, we note that since the average of $\rho$ is conserved, we shall not be able to find compact attractors on the whole space $H_{\mathrm{per}}^{1}(\Omega)$. We shall instead consider the restriction of $S(t)$ to closed subspaces of $H_{\mathrm{per}}^{1}(\Omega)$ of the form

$$
K_{\delta}=\left\{\rho \in H_{\text {per }}^{1}(\Omega),|m(\rho)| \leq \delta\right\}
$$

$\delta>0$, which are conserved by $S(t)$.

Now, our aim is to derive time uniform estimates.

First, we consider inequality (2.27). We note that the constant $c^{\prime \prime}$ in (2.27) depends on the constant $c(0)$ in (2.15) and tends to 0 as $c(0)$ tends to 0 . Furthermore, we note that since $|\rho|^{2} \leq c_{1} \int_{\Omega} \rho^{2 p+2} \mathrm{~d} x+c_{2}, c_{1}>0, c_{2} \geq 0$, we have for instance $\frac{1}{2} \int_{\Omega} \rho^{2 p+2} \mathrm{~d} x \geq \frac{1}{2 c_{1}}|\rho|^{2}-\frac{c_{2}}{2 c_{1}}$, and we deduce from (2.27) an inequality of the form

$$
\begin{aligned}
\frac{\mathrm{d}}{\mathrm{d} t}\left(\epsilon\|\bar{\rho}\|_{-1}^{2}+\epsilon \beta|\rho|^{2}+\alpha|\nabla \rho|^{2}+e^{2} \operatorname{Tr}(\mathbf{C} I)|\rho|^{2}+2 \int_{\Omega} f(\rho) \mathrm{d} x\right)+ & \epsilon \alpha|\nabla \rho|^{2}+c|\rho|^{2} \\
& +c^{\prime} \int_{\Omega} \rho^{2 p+2} \mathrm{~d} x+c^{\prime \prime}\left|\frac{\partial \rho}{\partial t}\right|^{2} \leq c^{\prime \prime \prime}|\rho|^{2}+c^{i v} .
\end{aligned}
$$


We note that we have not used the term $e^{2} \operatorname{Tr}(\mathbf{C} I)|\bar{\rho}|^{2}$ to derive (3.21). The reason is that we also wish to treat the case $e=0$. Furthermore, the constant $c^{\prime \prime \prime}$ in (3.21) tends to 0 as $c(0)$ tends to 0 and the constant $c^{i v}$ depends on $m\left(\rho_{0}\right)$ and is bounded independently of $m\left(\rho_{0}\right)$ if we restrict ourselves to $K_{\delta}$.

Then, we assume that $c-c^{\prime \prime \prime}>0$, where $c$ and $c^{\prime \prime \prime}$ are the constants in (3.21). This assumption is reasonable. Indeed, it can be achieved by choosing $\mathbf{C}$ and $e$ properly and, in particular, by taking $e$ small enough. Thus, we deduce from (3.21) an inequality of the form

$$
\begin{aligned}
\frac{\mathrm{d}}{\mathrm{d} t}\left(\epsilon\|\bar{\rho}\|_{-1}^{2}+\epsilon \beta|\rho|^{2}+\alpha|\nabla \rho|^{2}+e^{2} \operatorname{Tr}(\mathbf{C} I)|\rho|^{2}+2 \int_{\Omega} f(\rho) \mathrm{d} x\right)+c\left(\epsilon\|\bar{\rho}\|_{-1}^{2}+\epsilon \beta|\rho|^{2}+\alpha|\nabla \rho|^{2}\right. & \\
& \left.+e^{2} \operatorname{Tr}(\mathbf{C} I)|\rho|^{2}+c^{\prime} \int_{\Omega} \rho^{2 p+2} \mathrm{~d} x\right)+c^{\prime \prime}\left|\frac{\partial \rho}{\partial t}\right|^{2} \leq c^{\prime \prime \prime}
\end{aligned}
$$

where $c^{\prime}$ (and consequently $c$ ) will be fixed below. Now, we write that there exist $c_{3}$ and $c_{4}$ such that $\int_{\Omega} f(\rho) \mathrm{d} x \leq$ $c_{3} \int_{\Omega} \rho^{2 p+2} \mathrm{~d} x+c_{4}, c_{3}>0$. Taking the constant $c$ in (3.22) such that we can take $c^{\prime}=2 c_{3}$, we infer from (3.22) an inequality of the form

$$
\frac{\mathrm{d} E}{\mathrm{~d} t}+c E+c^{\prime}\left|\frac{\partial \rho}{\partial t}\right|^{2} \leq c^{\prime \prime}, c, c^{\prime}>0
$$

where $E=E(t)$ is defined in (2.28).

We deduce from (3.23) the existence of a bounded absorbing set for $S(t)$ on $K_{\delta}$.

Now, we deduce from (3.31), proceeding as in (2.32), an inequality of the form

$$
\frac{\mathrm{d}}{\mathrm{d} t}\left(|\rho|^{2}+\beta(B \nabla \rho, \nabla \rho)\right)+c\left|\nabla B^{\frac{1}{2}} \nabla \rho\right|^{2} \leq \zeta\left|\frac{\partial \rho}{\partial t}\right|^{2}+c^{\prime}\|\rho\|_{H_{\mathrm{per}}^{1}(\Omega)}^{2}+c^{\prime \prime}
$$

$\forall \zeta>0$. Summing (3.23) and (3.24), we find, if $\zeta$ is small enough

$$
\frac{\mathrm{d}}{\mathrm{d} t}\left(E+|\rho|^{2}+\beta(B \nabla \rho, \nabla \rho)\right)+c\left|\nabla B^{\frac{1}{2}} \nabla \rho\right|^{2} \leq c^{\prime}\|\rho\|_{H_{\mathrm{per}}^{1}(\Omega)}^{2}+c^{\prime \prime},
$$

and we deduce that there exists $t_{0}$ such that $t \geq t_{0}$ implies

$$
\int_{t}^{t+r}\|\rho\|_{H_{\mathrm{per}}^{2}(\Omega)}^{2} \mathrm{~d} s \leq c\left(t_{0}, r\right)
$$

Finally, we deduce from $(2.37,3.26)$ and the uniform Gronwall's Lemma (see [31]) the existence of a bounded absorbing set for $S(t)$ on $K_{\delta} \cap H_{\text {per }}^{2}(\Omega)$.

Thus, we have the

Theorem 3.1. We assume that $e$ is small enough and that $p=1$ or 2 when $n=3$. Then, the semigroup $S(t)$ associated with (2.16) possesses the global attractor $\mathcal{A}_{\delta}$ on $K_{\delta}$.

Now, our aim is to prove that $\mathcal{A}_{\delta}$ has finite dimension. Here, we cannot use the usual method based on the Lyapunov exponents (even when $b=0$; indeed, for technical reasons, we would anyway be obliged to work on spaces of the form

$$
\tilde{K}_{\delta}=\left\{\rho \in H_{\mathrm{per}}^{1}(\Omega), m(\rho)=\delta\right\}
$$

$\delta \in R$ (see [31]), which is not satisfactory). We shall instead use the results of Subsection 3.1. 
We set

$$
\begin{gathered}
L_{1} \rho=\rho-\beta \operatorname{div} B \nabla \rho, \\
L_{2} \rho=-b \cdot \nabla \rho, \\
A \rho=\frac{\alpha}{\beta} L_{1}(\rho-\Delta \rho), \\
\mathcal{R}(\rho)=-e^{2} \operatorname{Tr}(\mathbf{C} I) \operatorname{div} B \nabla \rho-\operatorname{div} B \nabla \mathcal{B}(\rho)-\operatorname{div} B \nabla f^{\prime}(\rho)+\frac{\alpha}{\beta} \Delta \rho-\frac{\alpha}{\beta} L_{1} \rho-\varphi .
\end{gathered}
$$

We easily prove that the assumptions made on $A, L_{1}$ and $L_{2}$ in Subsection 3.1 are satisfied (for $r=\frac{1}{4}$ ).

Then, we set

$$
X_{\delta}=\overline{\cup_{t \geq t_{1}} S(t) B_{2}}
$$

where $B_{2}$ is a bounded absorbing set for $S(t)$ on $K_{\delta} \cap H_{\text {per }}^{2}(\Omega)$ and $t_{1}$ is such that $t \geq t_{1}$ implies $S(t) B_{2} \subset B_{2}$. Thus, we deduce that $X_{\delta}$ is compact and positively invariant by $S(t)$. Finally, we prove, proceeding as in [24], that

$$
|\mathcal{R}(\rho)-\mathcal{R}(\bar{\rho})| \leq c\|\rho-\bar{\rho}\|_{H_{\text {per }}^{2}(\Omega)},
$$

$\forall \rho, \bar{\rho} \in X_{\delta}$.

In order to justify the formal estimates derived in Subsection 3.1, we note that since $X_{\delta}$ is bounded in $H_{\text {per }}^{2}(\Omega)$ and is positively invariant, it then follows from $(2.37)$ that $\rho(t) \in H_{\text {per }}^{2}(\Omega), \forall t \geq 0$, and that $\frac{\partial \rho}{\partial t}(t) \in H_{\text {per }}^{1}(\Omega)$, a.e. $t$. Furthermore, we have, if we take the scalar product of $(3.4)$ with $L_{1} w^{1}, w^{1}(t) \in H_{\text {per }}^{3}(\Omega)$, a.e. $t$. Therefore, $\frac{\mathrm{d} w^{1}}{\mathrm{~d} t}(t)=-L_{1}^{-1} A w^{1}(t)$ belongs to $H_{\mathrm{per}}^{1}(\Omega)$, a.e. $t$, which yields, if $\frac{\partial \rho}{\partial t}$ now corresponds to the difference of two trajectories, that $\frac{\mathrm{d} w^{2}}{\mathrm{~d} t}(t)$ belongs to $H_{\mathrm{per}}^{1}(\Omega)$, a.e. $t$, and thus to $D\left(L_{2}\right)$.

Thus, all the assumptions of Subsection 3.1 are satisfied. Furthermore, we easily prove that $S(t)$ is Lipschitz on $X_{\delta}$. This yields the

Theorem 3.2. The global attractor $\mathcal{A}_{\delta}$ obtained in Theorem 3.1 has finite fractal dimension.

Remark 3.2. Since the estimates derived above are also valid when $e=0$, we obtain the same results as in Theorems 3.1 and 3.2 for the semigroup associated with (2.9).

Remark 3.3. As a corollary, we obtain the existence of exponential attractors. Now, the problem of the existence of exponential attractors is an interesting problem on its own. Indeed, the global attractor has two major defaults for practical purposes. Indeed, it is very sensitive to perturbations and the rate of attraction of the trajectories can be arbitrarily small. An exponential attractor however, since it attracts exponentially the trajectories, will be more stable under perturbations. We refer the reader to [13] for a discussion on this subject.

Remark 3.4. When $g=0$, we can write (2.1) in the form

$$
\frac{\partial \rho}{\partial t}-\operatorname{div} B \nabla F\left(\rho, \frac{\partial \rho}{\partial t}\right)=0
$$

where

$$
F\left(\rho, \frac{\partial \rho}{\partial t}\right)=f^{\prime}(\rho)+\beta \frac{\partial \rho}{\partial t}-\alpha \Delta \rho+N^{-1} b . \nabla \frac{\partial \rho}{\partial t}-\gamma
$$


Taking the scalar product in $L^{2}(\Omega)$ of (3.33) with $F\left(\rho, \frac{\partial \rho}{\partial t}\right)$, we obtain (we assume that we have enough regularity)

$$
\frac{\mathrm{d}}{\mathrm{d} t} J(\rho)+\beta\left|\frac{\partial \rho}{\partial t}\right|^{2}+\left(B \nabla F\left(\rho, \frac{\partial \rho}{\partial t}\right), \nabla F\left(\rho, \frac{\partial \rho}{\partial t}\right)\right)=0,
$$

where

$$
J(\rho)=\int_{\Omega} f(\rho) \mathrm{d} x+\frac{\alpha}{2}|\nabla \rho|^{2}-\int_{\Omega} \gamma \rho \mathrm{d} x .
$$

We deduce that $J(\rho)$ is a Lyapunov function. We obtain a similar result when $\operatorname{div} B \nabla \gamma=g-b . \nabla g$ with $J(\rho)=\int_{\Omega} f(\rho) \mathrm{d} x+\frac{\alpha}{2}|\nabla \rho|^{2}$. The existence of a Lyapunov function, apart from its interest from the physical point of view, allows us to have more information on the structure of the global attractor, see [3] and [31] for more details. We note that we are not able to find a Lyapunov function when we take the deformations of the material into account (i.e. for $(2.16)$ when $\mathcal{B} \neq 0$ ).

\section{Attractors for the complete system}

We consider in this subsection equations (2.16) and (2.17). Our aim is to study the existence of finite dimensional attractors for the complete system (order parameter,displacement). To do so, we introduce the following family of operators:

$$
\mathcal{F}(t) \rho_{0}=(\rho(t), u(t))=(\rho(t), \mathcal{L}(\rho(t)))
$$

$t \geq 0$, where $\rho(t)=S(t) \rho_{0}$ is the solution of (2.16) with initial data $\rho_{0}$. We said in [7] that we have a weakly coupled system in the sense that the initial conditions for the two components are not independent; indeed, we have $u(0)=\mathcal{L}\left(\rho_{0}\right)$. We also note that the family of operators $\mathcal{F}(t)$ does not form a semigroup; therefore, we are not in the general (usual) framework for the study of attractors.

Let $\mathcal{A}_{\delta}$ be, as above, the global attractor for $S(t)$ on $K_{\delta}$. We set $\overline{\mathcal{A}}_{\delta}=\mathcal{L}\left(\mathcal{A}_{\delta}\right)$. Since $\mathcal{L}$ is continuous, $\overline{\mathcal{A}}_{\delta}$ is compact. Now, let $B_{0}$ be a bounded subset of $K_{\delta}$. Then, $\lim _{t \rightarrow+\infty} \operatorname{dist}\left(S(t) B_{0}, \mathcal{A}_{\delta}\right)=0$ (here, dist denotes the Hausdorff semi-distance), which yields, since $\mathcal{L}$ is Lipschitz, that

$$
\lim _{t \rightarrow+\infty} \operatorname{dist}\left(\mathcal{L} \circ S(t) B_{0}, \mathcal{L}\left(\mathcal{A}_{\delta}\right)\right)=0
$$

Thus, we deduce that

$$
\lim _{t \rightarrow+\infty} \operatorname{dist}\left(\mathcal{F}(t) B_{0}, \mathcal{A}_{\delta} \times \overline{\mathcal{A}}_{\delta}\right)=0
$$

We shall say that $\mathcal{A}_{\delta} \times \overline{\mathcal{A}}_{\delta}$ is an attractor for $\mathcal{F}(t)$ on $K_{\delta}$. Furthermore, since $\mathcal{A}_{\delta}$ has finite fractal dimension and since $\mathcal{L}$ is Lipschitz, we have $\operatorname{dim}_{F} \overline{\mathcal{A}}_{\delta} \leq \operatorname{dim}_{F} \mathcal{A}_{\delta}$ and $\operatorname{dim}_{F}\left(\mathcal{A}_{\delta} \times \overline{\mathcal{A}}_{\delta}\right) \leq 2 \operatorname{dim}_{F} \mathcal{A}_{\delta}$.

Now, we consider the restriction of $S(t)$ to $\tilde{K}_{\delta}$, where $\tilde{K}_{\delta}=\delta+\tilde{K}_{0}$ is defined in Section 2.2. Then, we have (see Sect. 1) $\mathcal{L}(\rho)=\overline{\mathcal{L}}(\rho-\delta)$ and $\mathcal{F}(t) \rho=(\delta, 0)+(S(t) \rho-\delta, \overline{\mathcal{L}}[S(t) \rho-\delta]), \forall \rho \in \tilde{K}_{\delta}$. Since $m(\rho)$ is conserved by $S(t)$, we can, without loss of generality, take $\delta=0$ and we consider the family of operators

$$
\begin{aligned}
\mathcal{F}(t): \tilde{K}_{0} & \rightarrow \tilde{K}_{0} \times \overline{\mathcal{L}}\left(\tilde{K}_{0}\right) \\
\rho_{0} & \mapsto\left(S(t) \rho_{0}, \overline{\mathcal{L}} \circ S(t) \rho_{0}\right),
\end{aligned}
$$

$t \geq 0$. Then, let $\mathcal{A}_{0}$ be the uniform attractor for $S(t)$ on $\tilde{K}_{0}\left(\mathcal{A}_{0}\right.$ is obtained exactly as in Section 3.2 and has finite fractal dimension). We can prove as above that $\mathcal{A}_{0} \times \overline{\mathcal{A}}_{0}$ is an attractor for $\mathcal{F}(t)$ on $\tilde{K}_{0}$ and that $\operatorname{dim}_{F}\left(\mathcal{A}_{0} \times \overline{\mathcal{A}}_{0}\right) \leq 2 \operatorname{dim}_{F} \mathcal{A}_{0}$, where $\overline{\mathcal{A}}_{0}=\overline{\mathcal{L}}\left(\mathcal{A}_{0}\right)$. Now, let us assume that $\mathbf{C} I$ is positive definite, in which case 
$\overline{\mathcal{L}}$ is bi-Lipschitz. We can prove (see [7]) that $\overline{\mathcal{A}}_{0}$ is the global attractor for the semigroup $\tilde{S}(t)=\overline{\mathcal{L}} \circ S(t) \circ \overline{\mathcal{L}}^{-1}$ on $\overline{\mathcal{L}}\left(\tilde{K}_{0}\right)$ and that $\mathcal{A}_{0} \times \overline{\mathcal{A}}_{0}$ is the global attractor for the semigroup $\tilde{\mathcal{F}}(t)=(S(t), \tilde{S}(t))$ on $\tilde{K}_{0} \times \overline{\mathcal{L}}\left(\tilde{K}_{0}\right)$. Furthermore, we have, in that case, $\operatorname{dim}_{F}\left(\mathcal{A}_{0} \times \overline{\mathcal{A}}_{0}\right)=2 \operatorname{dim}_{F} \mathcal{A}_{0}$.

Acknowledgements. The author wishes to thank an anonymous referee for many useful comments that helped improve the presentation of this article. He also wishes to thank Professor Mark I. Vishik for his interest in his work.

\section{REFERENCES}

[1] S. Agmon, A. Douglis and L. Nirenberg, Estimates near the boundary for solutions of partial differential equations satisfying general boundary conditions I, II. Comm. Pure Appl. Math. 12 (1959) 623-727; 17 (1964) 35-92.

[2] A. Babin and B. Nicolaenko, Exponential attractors of reaction-diffusion systems in an unbounded domain. J. Dyn. Differential Equations 7 (1995) 567-590.

[3] A.V. Babin and M.I. Vishik, Attractors of evolution equations. North-Holland, Amsterdam (1991).

[4] H. Brezis, Analyse fonctionnelle, théorie et applications. Masson (1983).

[5] J.W. Cahn, On spinodal decomposition. Acta Metall. 9 (1961) 795-801.

[6] J.W. Cahn and J.E. Hilliard, Free energy of a nonuniform system I. Interfacial free energy. J. Chem. Phys. 2 (1958) $258-267$.

[7] M. Carrive, A. Miranville, A. Piétrus and J.M. Rakotoson, The Cahn-Hilliard equation for an isotropic deformable continuum. Appl. Math. Letters 12 (1999) 23-28.

[8] M. Carrive, A. Miranville and A. Piétrus, The Cahn-Hilliard equation for deformable elastic continua. Adv. Math. Sci. Appl. (to appear).

[9] V.V. Chepyzhov and M. I. Vishik, Attractors of nonautonomous dynamical systems and their dimension. J. Math. Pures Appl. 73 (1994) 279-333.

[10] L. Cherfils and A. Miranville, Generalized Cahn-Hilliard equations with a logarithmic free energy (submitted).

[11] J.W. Cholewe and T. Dlotko, Global attractors of the Cahn-Hilliard system. Bull. Austral. Math. Soc. 49 (1994) $277-302$.

[12] A. Debussche and L. Dettori, On the Cahn-Hilliard equation with a logarithmic free energy. Nonlinear Anal. TMA 24 (1995) 1491-1514.

[13] A. Eden, C. Foias, B. Nicolaenko and R. Temam, Exponential attractors for dissipative evolution equations. Masson (1994).

[14] M. Efendiev and A. Miranville, Finite dimensional attractors for a class of reaction-diffusion equations in $\mathbb{R}^{n}$ with a strong nonlinearity. Disc. Cont. Dyn. Systems 5 (1999) 399-424.

[15] C.M. Elliot and S. Luckhauss, A generalized equation for phase separation of a multi-component mixture with interfacial free energy. Preprint.

[16] P. Fabrie and A. Miranville, Exponential attractors for nonautonomous first-order evolution equations. Disc. Cont. Dyn. Systems 4 (1998) 225-240.

[17] C. Galusinski, Perturbations singulières de problèmes dissipatifs : étude dynamique via l'existence et la continuité d'attracteurs exponentiels. Thèse, Université Bordeaux-I (1996).

[18] C. Galusinski, M. Hnid and A. Miranville, Exponential attractors for nonautonomous partially dissipative equations. Differential Integral Equations 12 (1999) 1-22.

[19] M. Gurtin, Generalized Ginzburg-Landau and Cahn-Hilliard equations based on a microforce balance. Physica D 92 (1996) $178-192$.

[20] J.L. Lions, Quelques méthodes de résolution des problèmes aux limites non linéaires. Dunod, Paris (1969).

[21] D. Li and C. Zhong, Global attractor for the Cahn-Hilliard system with fast growing nonlinearity. J. Differential Equations (1998).

[22] M. Marion and R. Temam, Navier-Stokes equations, theory and approximation, in Handbook of numerical analysis, P.G. Ciarlet and J.L. Lions eds. (to appear).

[23] A. Miranville, Exponential attractors for nonautonomous evolution equations. Appl. Math. Letters 11 (1998) 19-22.

[24] A. Miranville, Exponential attractors for a class of evolution equations by a decomposition method. C. R. Acad. Sci. 328 (1999) $145-150$.

[25] A. Miranville, Long time behavior of some models of Cahn-Hilliard equations in deformable continua. Nonlinear Anal. Series $B$ (to appear).

[26] A. Miranville, Exponential attractors for a class of evolution equations by a decomposition method. II. The nonautonomous case. C. R. Acad. Sci. 328 (1999) 907-912.

[27] A. Miranville, Equations de Cahn-Hilliard généralisées dans un milieu déformable. C. R. Acad. Sci. 328 (1999) $1095-1100$.

[28] A. Miranville, A model of Cahn-Hilliard equation based on a microforce balance. C. R. Acad. Sci. 328 (1999) $1247-1252$.

[29] A. Miranville, A. Piétrus and J.M. Rakotoson, Dynamical aspect of a generalized Cahn-Hilliard equation based on a microforce balance. Asymptotic Anal. 16 (1998) 315-345.

[30] B. Nicolaenko, B. Scheurer and R. Temam, Some global dynamical properties of a class of pattern formation equations. Comm. Partial Differential Equations 14 (1989) 245-297.

[31] R. Temam, Infinite dimensional dynamical systems in mechanics and physics. 2nd. ed., Springer-Verlag, New-York (1997). 